Journal of Engineering and Applied Sciences 15 (2): 430-436, 2020

ISSN: 1816-949X

(C) Medwell Journals, 2020

\title{
Design of an Insole using Force Sensing Resistors for Gait Analysis and Validation using Zebris FDM System
}

\author{
${ }^{1} \mathrm{R}$. Swarnalatha, ${ }^{2}$ Neelesh Kumar and ${ }^{3}$ Amrin Jameel Shanaz \\ ${ }^{1}$ Department of EEE, Birla Institute of Technology and Science, Pilani, Dubai Campus, \\ Dubai, United Arab Emirates \\ ${ }^{2}$ Principal Scientist, Biomedical Instrumentation Unit, CSIR-CSIO, Chandigarh, India \\ ${ }^{3}$ Department of ECE, Birla Institute of Technology and Science, Pilani, Dubai Campus, \\ Dubai, United Arab Emirates
}

\begin{abstract}
In this study, we present an insole with three Force Sensing Resistors (FSR) one each for toe, hallux and heel which provides quantitative gait analysis outside the confinement of traditional lab at a low cost. The aim of the study was to explore the validity and reliability of the insole in a healthy individual. Gait data were collected from a healthy individual at three different paces of walking. Validation of the data was performed with zebris FDM system. Through a series of analytical tests we tested the accuracy and validated the performance of FSR insole. With the future scope being, developing an algorithm for a smartphone app that acquires the acceleration signal from the insole for users to assess the abnormality of gait.
\end{abstract}

Key words: Gait analysis, Force Sensing Resistors (FSRs), FDM-S system (zebris), ANOVA test, t-test, Bland-Altman plot

\section{INTRODUCTION}

Gait Analysis (GA) is an important diagnostic measure to investigate the pattern of walking to characterize human locomotion (Whittle, 1996; Johansson, 1975). Traditional gait analysis is generally, carried out in a gait lab with equipped force and body tracking sensors which needs a trained medical professional to interpret the results. This procedure is tedious, expensive and unfeasible. It has been widely used in various applications such as medical diagnostics, rehabilitation and sports (Li et al., 2009; Bianchi et al., 2010; Baker, 2006; Huang et al., 2014; Xu et al., 2012a, b).

Some typical examples are the use of conducting foot switches (Zijlstra et al., 1995; Nevill et al., 1995), pressure-sensitive foot switches (Eastlack et al., 1991), dynamometric platforms (Nilsson and Thorstensson, 1987) or stereo-photogrammetric systems (Zijlstra and Dietz, 1995) that are the traditional and more reliable methods to assess the spatio-temporal gait parameters and the several gait phases. The GA performed with biomechanical and foot-pressure sensors is used to assess and if necessary, correct the gait and posture of an athlete or an injured person alike (Klenerman et al., 1988; Ounpuu, 1994; Zatsiorky et al., 1994). Geriatrics makes extensive use of GA to assess and predict the risks associated with mobility in the elderly (Rodgers, 1995;
Wolfson et al., 1990; Berg et al., 1992; Wrisley and Kumar, 2010). One such method to analyze gait is based on the multi-camera motion capture system and a platform which is capable of measuring ground-reaction forces (Patrick and Keenan, 2007; Aminian et al., 2002). When the subject/patient walks along the platform, his motion patterns are captured by the camera and the sensors embedded onto the floor (Salarian et al., 2004; Moore et al., 2007). However, this type of motion capture system is usually restricted to indoor use as motion capture camera often rely on infrared reflective markers that will be affected by sunlight. The high cost of the equipment also makes gait analysis prohibitive for wide adoption. Moreover, using the data captured by these devices, the trained expert needs to be analyzed by trained experts manually (Zijlstra, 2004).

With the development of wearable sensors, gait analysis becomes quite inexpensive and the monitoring can be performed in comfortable and realistic settings, outside the lab. There has been prior research on using sensors like accelerometers and gyroscopes to analyze various gait features (Dejnabadi et al., 2006). Bae et al. 2009 designed a Force Sensing Resistive (FSR) sensor array based gait analysis and Chen et al. (2011) proposed a longitudinal motion assessment method using inertial sensors on cerebral palsy patients Liu et al. (2011). Researchers have been working on designing a pair of

Corresponding Author: R. Swarnalatha, Department of EEE, Birla Institute of Technology and Science, Pilani, Dubai Campus, Plot No.UG 06, P.O. Box 345055, Dubai International Academic City, Dubai, United Arab Emirates 
instrumented insoles with piezoresistive pressure sensor and three-axis accelerometers which can measure, pressure distribution of the foot during various gait phases (Martinez-Marti et al., 2014). Recently, Xu et al. (2012a, b) proposed an insole which consists an array of 48 pressure and motion sensing sensors to analyze gait parameters. Although, these methods help us understand the walking pattern efficiently, they cannot address the problem of analyzing gait cycle and its features accurately in real-time studies of foot pressure measurement and application of insole sensor in measuring plantar pressure was published.

Gait analysis is an important tool to detect fall risk and fall prevention in ageing population. The wearable gait analysis system, mentioned in this paper can analyze gait parameters such as stance phase, load response, mid stance, pre-swing. Swing phase, double stance phase stride time, step time and cadence step/min by analyzing pressure between the foot plantar surface and the shoe insole.

This insole can be used to study the gait parameters in Parkinson's patients. Parkinson's Disease (PD) is a chronic and progressive hypokinetic disorder of the CNS caused by basal ganglia dysfunction. Four major motor symptoms of PD are resting tremor of 4-6 $\mathrm{Hz}$ (the most manifest symptom), rigidity (stiffness in muscles), bradykinesia (slow physical movement), postural instability (loss of postural reflexes) (Xu et al., $2012 a, b)$ ). Other symptoms may include physical fatigue, festination, small shuffling steps and decreases in both arm swing and walking speed. The research group led by Jankovic (2008), Blin et al. (1990) and Sekine et al. (2002). Applied the wavelet-based fractal analysis and the time-frequency matching pursuit algorithm to the acceleration signals recorded from PD subjects during climbing stairs and walking along a corridor. In Parkinson's disease, SVM has been used to estimate the severity of tremor, bradykinesia and dyskinesia (Patel et al., 2009). Studies of gait in PD using SVMs have been limited to plantar pressure data (Jeon et al., 2008) and ground reaction forces (Yang et al., 2009).

\section{MATERIALS AND METHODS}

\section{System overview}

Framework: Identifying normal and pathological walking patterns is a complicated process and requires analysis of various gait features like cadence, stride height, stride length and speed which are very basic features. In that way, gait is a cycle movement which can be categorized by two main phases: stance phase and the swing phase. The first corresponds to the period of time in which the foot is in contact with the ground and it starts when the heel first strikes the floor, lasting until the moment the toe becomes the last contact point (toe off). The swing phase, on the other hand, represents the lack of contact with the floor and it starts when the toe ceases to

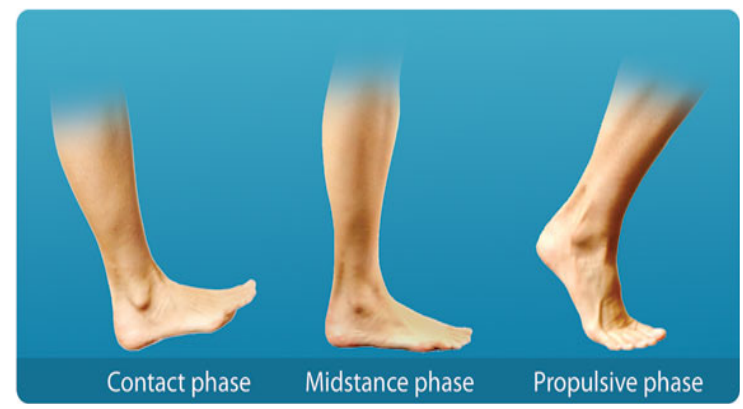

Fig. 1: Three phases in gait cycle

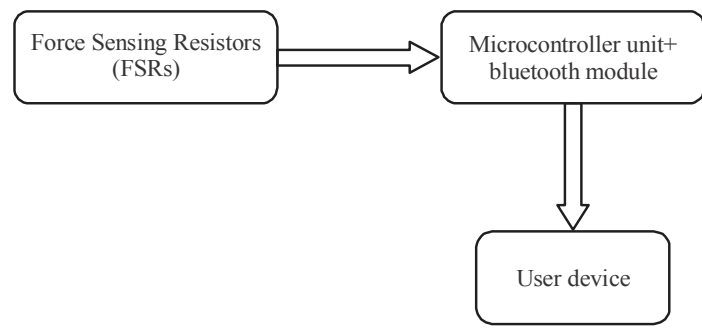

Fig. 2: Overview of the mechanism

be in contact with the ground, persisting till the moment the heel strikes the floor again, then starting a new gait cycle. For an individual with no abnormalities, the stance phase (at a normal pace) corresponds to about $\sim 62 \%$ of the entire gait cycle (Klenerman et al., 1988; Moore et al., 2007).

In context to medical application, each gait cycle is divided into three phases: contact, mid stance and propulsive phase as shown in Fig. 1.

Our main goal is to design an insole which is capable of identifying the gait stage in real-time and detect various gait parameters, required to analyze human gait. To address this, we came up with an overall system design which is divided into three major parts, the insole hardware design, data acquisition and transmission using Bluetooth module and finally the data processing and graphical user interface as shown in Fig. 2.

Insole: The insole has 3 FSRs one each for toe, hallux (first metatarsal) and heel as shown in Fig. 3. The FSRs are placed at toe, first metatarsal or hallux and heel of the foot. These points are chosen because of the natural anatomy of the foot as they face the most pressure during a gait cycle. The prototype has sensor inlayed insole, a Microcontroller Unit (MCU): Arduino nano with: pull-down resistors and header pins to connect $\mathrm{MCU}$ and other components, a Bluetooth module: HC-05 (for wireless transmission of data) and $5 \mathrm{~V}$ power for each foot. The block diagram of the connection is shown in Fig. 4. 
(a)

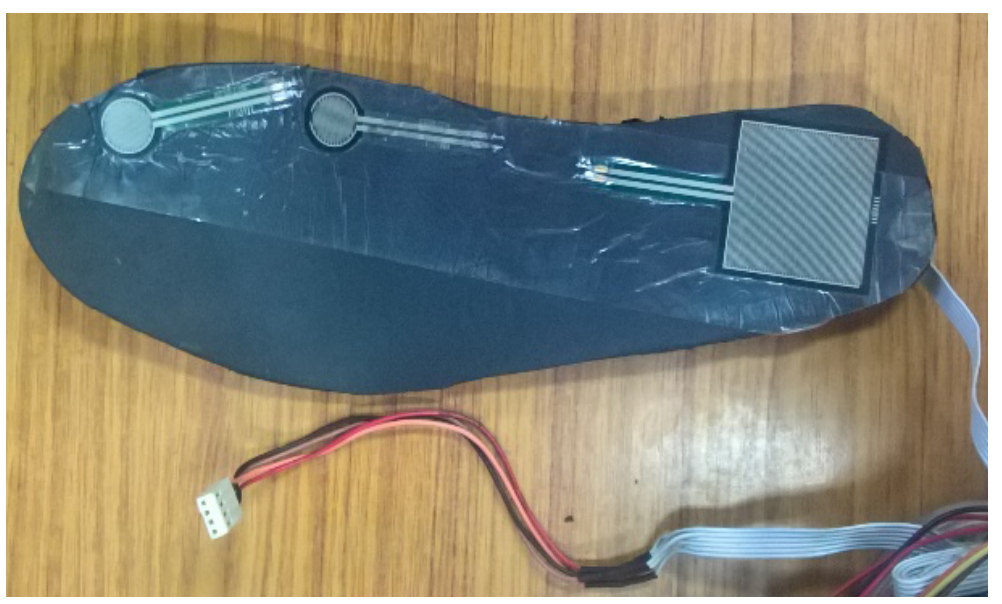

(b)

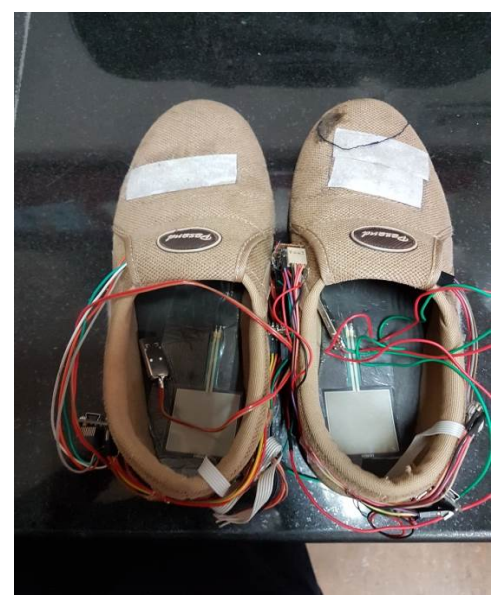

Fig. 3(a-b): Insole with positioning of the 3 FSRs (toe, ball, heel)

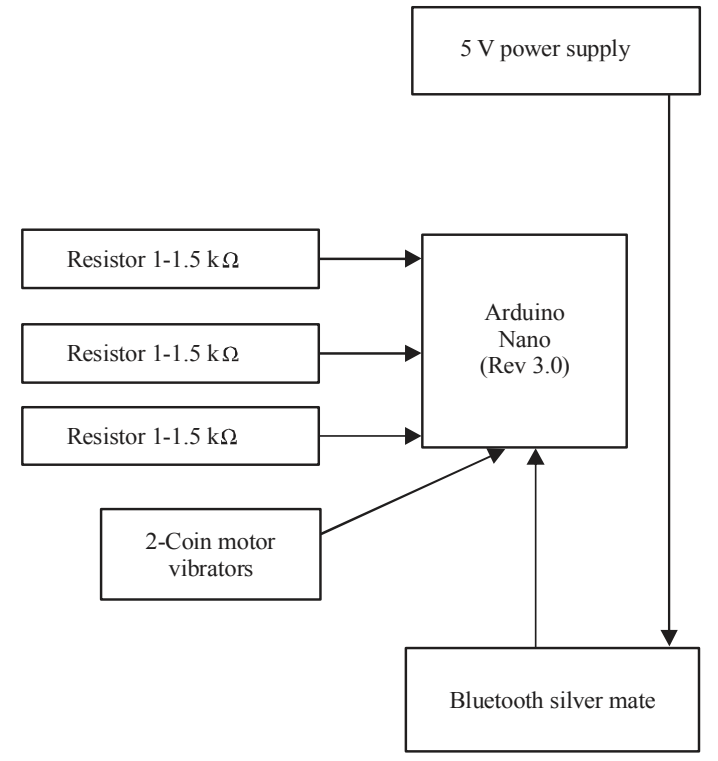

Fig. 4: Block diagram of the circuit connection

Experimental protocol: The subject performed 10 walks at different paces: normal, fast and slow paces. Each walk lasted for $3 \mathrm{~min}$. The validity of the gait parameters measured was tested against FDM-S pressure plate as shown in Fig. 5.

The zebris measuring system due to the very large measuring areas of $144 \times 56,208 \times 56$ and $304 \times 56 \mathrm{~cm}$, the dynamic force and pressure distribution under the feet can be recorded from several contacts with the ground and analyzed (Yang et al., 2009; Brunner, 2011). Many previous studies have used zebris as the benchmark (Brunner, 2014; Rueterbories et al., 2010; Romkes et al., 2005).
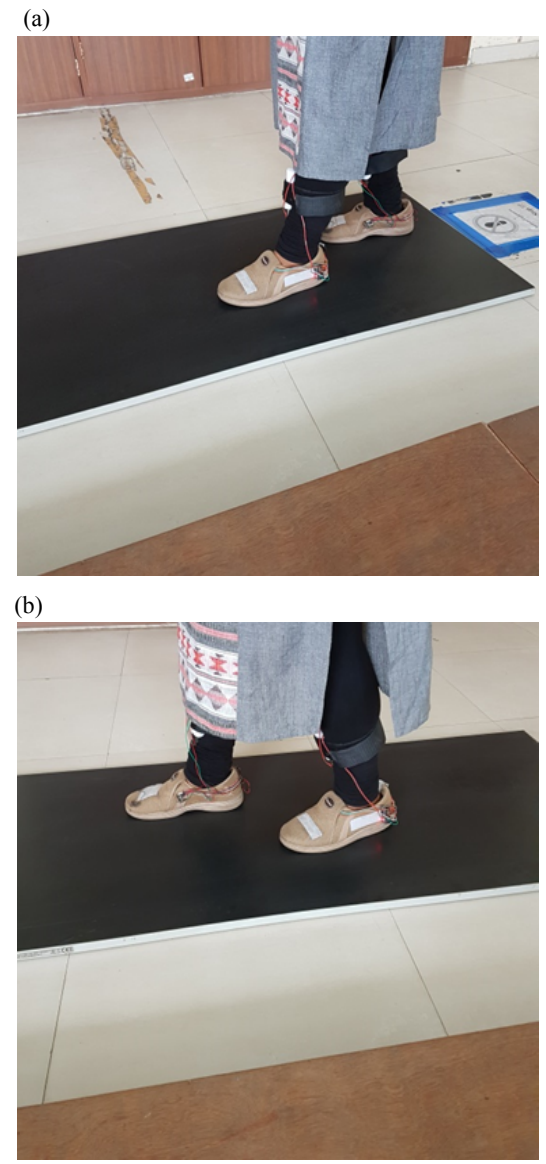

Fig. 5(a-b): Experimental setup

\section{RESULTS AND DISCUSSION}

Statistical analysis: Mean of every gait parameter were calculated at each pace and for every trial. ANOVA-2 
J. Eng. Applied Sci., 15 (2): 430-436, 2020

Table 1: Gait parameter comparison-normal walk

\begin{tabular}{lcccc}
\hline Phases & Zebris gait & FSR gait & Error & Error (\%) \\
\hline Stance phase L (\%) & 66.40 & 67.60 & 1.20 & 1.70 \\
Stance phase R (\%) & 65.50 & 66.20 & 0.70 & 1.05 \\
Load response (\%) & 16.00 & 16.41 & 0.41 & 2.40 \\
Mid stance R (\%) & 33.60 & 32.70 & 0.90 & 2.70 \\
Pre -swing L (\%) & 16.00 & 16.40 & 0.40 & 2.40 \\
Pre-swing R (\%) & 16.00 & 16.50 & 0.50 & 3.00 \\
Swing phase L (\%) & 33.60 & 32.80 & 0.80 & 3.40 \\
Swing phase R (\%) & 34.50 & 33.90 & 0.60 & 1.70 \\
Double stance phase (\%) & 31.90 & 32.40 & 0.50 & 1.50 \\
Stride time (sec) & 1.19 & 1.26 & 0.07 & 5.50 \\
Step time L (sec) & 0.59 & 0.61 & 0.02 & 3.20 \\
Step time R (sec) & 0.60 & 0.65 & 0.05 & 7.60 \\
Cadence (step/min) & 101.00 & 95.00 & 6.00 & 6.30 \\
\hline
\end{tabular}

Table 2: Gait parameter comparison-slow walk

\begin{tabular}{lcccc}
\hline Phases & Zebris gait & FSR gait & Error & Error (\%) \\
\hline Stance phase L (\%) & 65.10 & 66.40 & 1.30 & 1.90 \\
Stance phase R (\%) & 66.70 & 67.10 & 0.40 & 0.50 \\
Load response (\%) & 16.30 & 16.80 & 0.50 & 2.90 \\
Mid stance R (\%) & 34.90 & 34.30 & 0.60 & 1.70 \\
Pre-swing L (\%) & 16.30 & 16.80 & 0.50 & 2.90 \\
Pre-swing R (\%) & 15.50 & 15.99 & 0.49 & 3.00 \\
Swing phase L (\%) & 34.90 & 33.90 & 1.00 & 2.90 \\
Swing phase R (\%) & 33.30 & 33.10 & 0.20 & 0.60 \\
Double stance phase (\%) & 31.80 & 32.50 & 0.70 & 2.10 \\
Stride time (sec) & 1.29 & 1.39 & 0.10 & 7.19 \\
Step time L (sec) & 0.66 & 0.70 & 0.04 & 5.71 \\
Step time R (sec) & 0.63 & 0.69 & 0.06 & 8.60 \\
Cadence (step/min) & 93.00 & 86.00 & 7.00 & 8.13 \\
\hline
\end{tabular}

Table 3: Gait parameter comparison-fast walk

\begin{tabular}{lcccc} 
Table 3: Gait parameter comparison-fast walk \\
\hline Phases & Zebris gait & FSR gait & Error & Error (\%) \\
\hline Stance phase L (\%) & 65.7 & 66.20 & 0.50 & 0.70 \\
Stance phase R (\%) & 63.8 & 64.30 & 0.50 & 0.70 \\
Load response (\%) & 15.2 & 15.80 & 0.60 & 3.70 \\
Mid stance R (\%) & 34.3 & 34.80 & 0.50 & 1.43 \\
Pre-swing L (\%) & 15.2 & 15.90 & 0.70 & 4.40 \\
Pre-swing R (\%) & 14.3 & 14.77 & 0.47 & 3.18 \\
Swing phase L (\%) & 34.3 & 34.10 & 0.20 & 0.58 \\
Swing phase R (\%) & 36.2 & 35.90 & 0.30 & 0.83 \\
Double stance phase (\%) & 29.5 & 30.20 & 0.70 & 2.31 \\
Stride time (sec) & 1.05 & 1.12 & 0.07 & 6.25 \\
Step time L (sec) & 0.52 & 0.55 & 0.03 & 5.45 \\
Step time R (sec) & 0.53 & 0.57 & 0.04 & 7.01 \\
Cadence (step/min) & 114.0 & 107.00 & 7.00 & 6.50 \\
\hline
\end{tabular}

Table 4: Stance phase

\begin{tabular}{lcc}
\hline Stance phase (walk) & FSR (\%) & Zebris (\%) \\
\hline Normal & 0.676 & 0.664 \\
Slow & 0.664 & 0.651 \\
Fast & 0.662 & 0.657 \\
\hline
\end{tabular}

Table 5: ANOVA-2 factor for stance phase

\begin{tabular}{lllll}
\hline Source of variation & df & F & p-values & F crit \\
\hline Rows & 2 & 9.491228 & 0.095318 & 19 \\
Columns & 1 & 15.78947 & 0.057889 & 18.51282 \\
\hline
\end{tabular}

Table 6: F-test two-sample for stance phase left

\begin{tabular}{lll}
\hline Parameters & FSR gait & Zebris gait \\
\hline Mean & 66.73333 & 65.73333 \\
Variance & 0.573333 & 0.423333 \\
Observations & 3 & 3 \\
df & 2 & 2 \\
\hline F: $1.354331, \mathrm{P}(\mathrm{F}<=$ f) one-tail: 0.424749, F: Critical one-tail 19
\end{tabular}

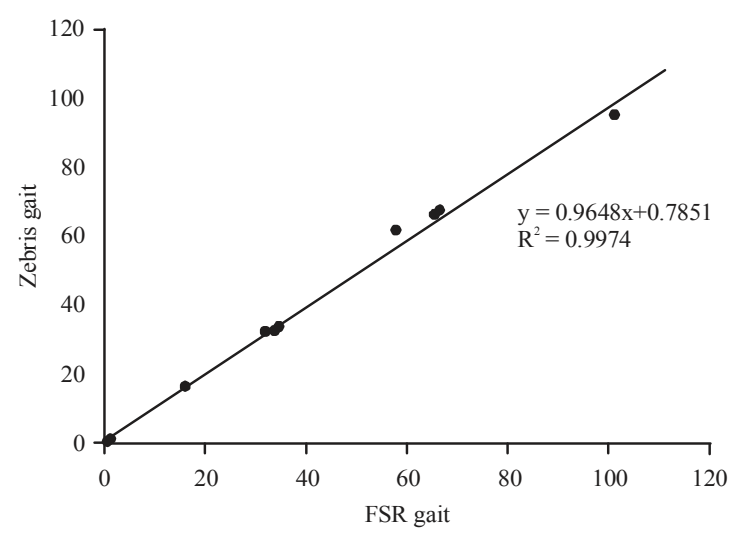

Fig. 6: Two factor ANOVA without replication data analysis tool

Table 7: The t-test: two-sample assuming unequal variances

\begin{tabular}{lll}
\hline Parameters & Zebris gait & FSR gait \\
\hline Mean & 32.06769231 & 31.725385 \\
Variance & 897.3112192 & 837.47333 \\
df & 24 & \\
\hline t stat: $0.029632314, \mathrm{P}(\mathrm{T}<=\mathrm{t})$ & one-tail: $0.488302668, \mathrm{t}$ critical one-tail: \\
$1.71088208, \mathrm{P} \quad(\mathrm{T}<=\mathrm{t})$ & two-tail: $0.976605335, \mathrm{t}$ critical two-tail: \\
2.063898562 &
\end{tabular}

factor without replication test (Wearing et al., 2013) were performed to compare the gait parameters measured by FSRs and zebris as shown in Table 1-4.

Table 5 has two parts. The first part provides statistics for the rows. The second part provides statistics for the columns. $\mathrm{df}$ is the degrees of freedom. Degrees of freedom (n-1) of an estimate isthe number of independent pieces of information that went into calculating the estimate. The table features two values for F. One F is for the rows and the other is for the columns. The p-value is the proportion of area that the $\mathrm{F}$ cuts off in the upper tail of the F-distribution. If this value is $<0.05$, reject null hypothesis. Along the columns $15.789<18.512$, $\left(\mathrm{F}<\mathrm{F}_{\text {critical }}\right) \mathrm{p}>0.05$, we can't reject the null hypothesis and indicating no statistical difference between the performance in FSR and zebris. Figure 6 shows the two factor ANOVA without replication data analysis tool (Reed III, 2003).

The ANOVA test was performed to gait parameters of the three trials. There were no significant differences in other gait parameters. Table 6 shows the F test two sample for stance phase left F-test was performed to test if the null hypothesis between the variances of two systems are equal. Since, $F<F_{\text {critical }}$ we do not reject the null hypothesis which indicates the variances of the two populations are equal.

The t-test (Pandey, 2015; Jiaxi, 2010) was performed on all the parameters measured. Table 7 shows, the t-test for two-sample assuming unequal variances. Using one-tailed test, we are testing for the possibility of the relationship in one direction and completely disregarding the possibility of a relationship in the other direction. Our null hypothesis is that the zebris gait mean 


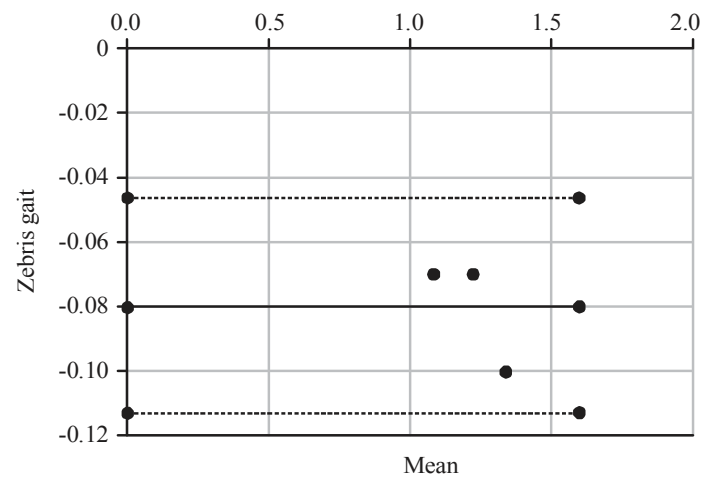

Fig. 7: Bland-Altman plot for stride time

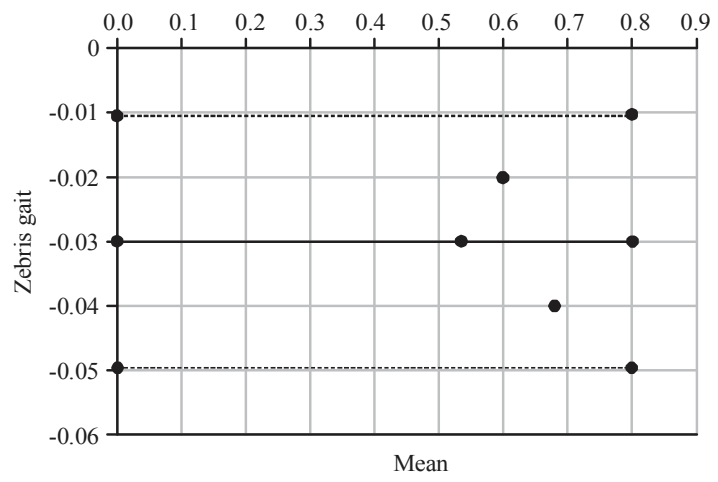

Fig. 8: Bland-Altman plot for stance (L) time

is not equal to FSR gait say $\mathrm{x}$. A one-tailed test will test either if the mean is significantly greater than $\mathrm{x}$ or if the mean is significantly less than $\mathrm{x}$ but not both. Then, depending on the chosen tail, the mean is significantly greater than or less than $\mathrm{x}$ if the test statistic is in the top $5 \%$ of its probability distribution or bottom $5 \%$ of its probability distribution, resulting in a $\mathrm{p}<0.05$, following which if $\mathrm{t}$ stat $<-\mathrm{t}$ Critical two-tail or $\mathrm{t} s \mathrm{sta}>\mathrm{t}_{\text {Critical }}$ two-tail, we reject the null hypothesis. This is not the case, $-2.063<0.029<2.063$. Therefore, we do not reject the null hypothesis where null hypothesis signifies no similarity between zebris and FSR gait. The observed difference between the sample means (32-31.7) is convincing enough to say that the gait parameters between FSR and zebris do not differ significantly.

Validity: Correlation scatterplot (regression line) was plotted for various gait parameters measured by zebris and FSR which depicts correlation between gait parameters measure by zebris and FSR. High correlation coefficient $R^{2}$ indicates a high correlation. The scatter plot shows a positive or direct association between zebris and FSR gait measurements.
Bland-Altman plot (Giavarina, 2015; Altman and Bland, 2017) is used as a comparison test in measurement of same variable by two setups, zebris and FSR. The Bland-Altman plot shown in Fig. 7 and 8 shows that all the values of stride time for normal, fast and slow walk both in zebris and FSR lie between limits of agreement $(-0.08)$ and the error margins. Intra class correlation for stance time in seconds was 0.8162 .

Furthermore, there was no statistical difference between the insole and the force plate with regards to the resulting force and stance time. The Bland-Altman plots confirm the validation results, showing equal error margins between the limits of agreement. The standard deviations and variances are also within these limits, further confirming the insole's validity.

\section{CONCLUSION}

The monitoring of gait is important to improve the quality of care for PD patients. Many active researches have been attempting to do it objectively and autonomously. Unlike (Martinez-Marti et al., 2014; Xu et al., 2012a, b; Dugan and Bhat, 2005) we just use 3 force sensing resistors to carry real-time gait analysis, achieving long battery life, making it unique for various application likes sports, entertainment and rehabilitation. Our proposed methodology focuses on unobtrusive gait analysis detection by using a FSRs embed insole to improve usability. In terms of clinical applications, the insole can be used to detect the gait analysis results which can be transmitted to a centralized medical location and processed by trained medical personnel remotely.

\section{RECOMMENDATIONS}

The treatment and rehabilitation of patients cannot occur until there is a diagnosis of how disabled a patient is. The coin vibration motor present in the insole can be provide vibrations in SYNC with phases of gait, for rehabilitation therapy. The future scope is to design and develop gait detection algorithm in smartphones that acquires the acceleration signal from the insole for users to assess the abnormality of gait. The accelerometer in the smartphone can be used to measure gait characteristics. In the future, this smartphone app will show the gait abnormality and be able to use as a portable rehabilitation device in the clinic and possibly at home.

\section{ACKNOWLEDGEMENT}

The authors would like to thank Prof. R.N. Saha, Director, BITS, Pilani -Dubai Campus for his constant encouragement and support. 


\section{REFERENCES}

Altman, D.G. and J.M. Bland, 2017. Assessing agreement between methods of measurement. Clin. Chem., 63: 1653-1654.

Aminian, K., B. Najafia, C. Bülab, P.F. Leyvrazc and P. Robert, 2002. Spatio-temporal parameters of gait measured by an ambulatory system using miniature gyroscopes. J. Biomech., 35: 689-699.

Bae, J., K. Kong, N. Byl and M. Tomizuka, 2009. A mobile gait monitoring system for gait analysis. Proceedings of the 2009 IEEE International Conference on Rehabilitation Robotics, June 23-26, 2009, IEEE., Kyoto, Japan, pp: 73-79.

Baker, R., 2006. Gait analysis methods in rehabilitation. J. NeuroEng. Rehabil., Vol. 3. 10.1186/1743-0003-3-4.

Berg, K.O., B.E. Maki, J.I. Williams, P.J. Holliday and S.L. Wood-Dauphinee, 1992. Clinical and laboratory measures of postural balance in an elderly population. Arch. Phys. Med. Rehabil., 73: 1073-1080.

Bianchi, F., S.J. Redmond, M.R. Narayanan, S. Cerutti and N.H. Lovell, 2010. Barometric pressure and triaxial accelerometry-based falls event detection. IEEE. Trans. Neural Syst. Rehabil. Eng., 18: 619-627.

Blin, O., A.M. Ferrandez and G. Serratrice, 1990. Quantitative analysis of gait in Parkinson patients: Increased variability of stride length. J. Neurol. Sci., 98: 91-97.

Brunner, W., 2011. Gait analysis apparatus and method using a treadmill. United States Patent No. US8002672B2.

Brunner, W., 2014. Gait analysis system. United States Patent No. US8790279B2.

Chen, S., C.L. Cunningham, B.C. Bennett and J. Lach, 2011. Enabling longitudinal assessment of ankle-foot orthosis efficacy for children with cerebral palsy. Proceedings of the 2nd International Conference on Wireless Health (WH '11), October 10-13, 2011, ACM, San Diego, California, USA., ISBN: 978-1-4503-0982-0, pp: 1-4.

Dejnabadi, H., B.M. Jolles, E. Casanova, P. Fua and K. Aminian, 2006. Estimation and visualization of sagittal kinematics of lower limbs orientation using body-fixed sensors. IEEE. Trans. Biomed. Eng., 53: 1385-1393.

Dugan, S.A. and K.P. Bhat, 2005. Biomechanics and analysis of running gait. Phys. Med. Rehabil. Clin. North Am., 16: 603-621.

Eastlack, M.E., J. Arvidson, L. Snyder-Mackler, J.V. Danoff and C.L. McGarvey, 1991. Interrater reliability of videotaped observational gait-analysis assessments. Phys. Ther., 71: 465-472.
Giavarina, D., 2015. Understanding bland altman analysis. Biochem. Med. (Zagreb), 25: 141-151.

Huang, M.C., S.H. Lee, S.C. Yeh, R.C. Chan and A. Rizzo et al., 2014. Intelligent frozen shoulder rehabilitation. IEEE. Intell. Syst., 29: 22-28.

Jankovic, J., 2008. Parkinson's disease: Clinical features and diagnosis. J. Neurol. Neurosurg. Psychiatry, 79: 368-376.

Jeon, H.S., J. Han, W.J. Yi, B. Jeon and K.S. Park, 2008. Classification of Parkinson gait and normal gait using spatial-temporal image of plantar pressure. Proceedings of the 30th Annual International Conference on the IEEE Engineering in Medicine and Biology Society, August 20-25, 2008, IEEE., Vancouver, Canada, pp: 4672-4675.

Jiaxi, L., 2010. The application and research of T-test in medicine. Proceedings of the 1st International Conference on Networking and Distributed Computing, October 21-24, 2010, IEEE., Hangzhou, China, pp: 321-323.

Johansson, G., 1975. Visual motion perception. Scient. Am., 232: 76-89.

Klenerman, L., R.J. Doses, C. Weller, A.L. Leeman and P.W. Nicholson, 1988. Bringing gait analysis out of the laboratory and into the clinic. Age Ageing, 17: 397-400.

Li, Q., J.A. Stankovic, M.A. Hanson, A.T. Barth, J. Lach and G. Zhou, 2009. Accurate, fast fall detection using gyroscopes and accelerometer-derived posture information. Proceedings of the 6th International Workshop on Wearable and Implantable Body Sensor Networks, June 3-5, 2009, IEEE., Berkeley, California, USA., pp: 138-143.

Liu, T., Y. Inoue and K. Shibata, 2009. Development of a wearable sensor system for quantitative gait analysis. Measurement, 42: 978-988.

Martinez-Marti, F., M.S. Martinez-Garcia, S.G. Garcia-Diaz, J. Garcia-Jimenez, A.J. Palma and M.A. Carvajal, 2014. Embedded sensor insole for wireless measurement of gait parameters. Australas. Phys. Eng. Sci. Med., 37: 25-35.

Moore, S.T., H.G. MacDougall, J.M. Gracies, H.S. Cohen and W.G. Ondo, 2007. Long-term monitoring of gait in Parkinson's disease. Gait Posture, 26: 200-207.

Nevill, A.J., M.G. Pepper and M. Whiting, 1995. In-shoe foot pressure measurement system utilising piezoelectric film transducers. Med. Biol. Eng. Comput., 33: 76-81.

Nilsson, J. and A. Thorstensson, 1987. Adaptability in frequency and amplitude of leg movements during human locomotion at different speeds. Acta Physiol. Scand., 129: 107-114.

Ounpuu, S., 1994. The biomechanics of walking and running. Clin. Sports Med., 13: 843-863. 
Pandey, R.M., 2015. Commonly used T-tests in medical research. J. Pract. Cardiovasc. Sci., 1: 185-188.

Patel, S., K. Lorincz, R. Hughes, N. Huggins and J. Growdon et al., 2009. Monitoring motor fluctuations in patients with Parkinson's disease using wearable sensors. IEEE. Trans. Inf. Technol. Biomed., 13: 864-873.

Patrick, J.H. and M.A.E. Keenan, 2007. Gait analysis to assist walking after stroke. Lancet, 369: 256-257.

Reed III, J.F., 2003. Analysis of variance (ANOVA) models in emergency medicine. Internet J. Emerg. Intensive Care Med., Vol. 7, No. 2.

Rodgers, M.M., 1995. Dynamic foot biomechanics. J. Orthop. Sports. Phys. Ther., 21: 306-316.

Romkes, J., C. Rudmann and R. Brunner, 2005. Changes in gait and EMG when walking with the Masai Barefoot Technique. Clin. Biomech., 21: 75-81.

Rueterbories, J., E.G. Spaich, B. Larsen and O.K. Andersen, 2010. Methods for gait event detection and analysis in ambulatory systems. Med. Eng. Phys., 32: 545-552.

Salarian, A., H. Russmann, F.J.G. Vingerhoets, C. Dehollain, Y. Blanc, P.R. Burkhard and K. Aminian, 2004. Gait Assessment in Parkinson's Disease: Toward an Ambulatory System for Long-Term Monitoring. IEEE Trans. Biomed. Eng., 51: 1434-1443.

Sekine, M., M. Akay, T. Tamura, Y. Higashi and T. Fujimoto, 2004. Fractal dynamics of body motion in patients with Parkinson's disease. J. Neural Eng., 1: $8-15$

Sekine, M., T. Tamura, M. Akay, T. Fujimoto, T. Togawa and Y. Fukui, 2002. Discrimination of walking patterns using wavelet-based fractal analysis. IEEE Trans. Neural Syst. Rehabil. Eng., 10: 188-196.

Wearing, S.C., L.F. Reed and S.R. Urry, 2013. Agreement between temporal and spatial gait parameters from an instrumented walkway and treadmill system at matched walking speed. Gait Posture, 38: 380-384.

Whittle, M.W., 1996. Clinical gait analysis: A review. Hum. Mov. Sci., 15: 369-387.
Wolfson, L., R. Whipple, P. Amerman and J.N. Tobin, 1990. Gait assessment in the elderly: A gait abnormality rating scale and its relation to falls. J. Gerontol., 45: M12-M19.

Wrisley, D.M. and N.A. Kumar, 2010. Functional gait assessment: Concurrent, discriminative and predictive validity in community-dwelling older adults. Phys. Ther., 90: 761-773.

Xu, W., M.C. Huang, N. Amini, J.J. Liu, L. He and M. Sarrafzadeh, 2012a. Smart insole: A wearable system for gait analysis. Proceedings of the 5th International Conference on PErvasive Technologies Related to Assistive Environments, June 6-8, 2012, ACM., Heraklion, Crete, Greece, pp: 1-4.

Xu, X., M. Batalin, Y. Wang and W. Kaiser, 2012b. Gait quality evaluation method for post-stroke patients. Proceedings of the 2012 IEEE International Conference on Acoustics, Speech and Signal Processing (ICASSP), March 25-30, 2012, IEEE., Kyoto, Japan, pp: 613-616.

Yang, M., H. Zheng, H. Wang and S. McClean, 2009. Feature selection and construction for the discrimination of neurodegenerative diseases based on gait analysis. Proceedings of the 3rd International Conference on Pervasive Computing Technologies for Healthcare, April 1-3, 2009, IEEE., London, England, UK., pp: 1-7.

Zatsiorky, V.M., S.L. Werner and M.A. Kaimin, 1994. Basic kinematics of walking step length and step frequency: A review. J. Sports Med. Phys. Fitness, 34: 109-134.

Zijlstra, W. and V. Dietz, 1995. Adaptability of the human stride cycle during split-belt walking. Gait Posture, 3: 250-257.

Zijlstra, W., 2004. Assessment of spatio-temporal parameters during unconstrained walking. Eur. J. Applied Physiol., 92: 39-44.

Zijlstra, W., A.W.F. Rutgers, A.L. Hof and T.W. van Weerden, 1995. Voluntary and involuntary adaptation of walking to temporal and spatial constraints. Gait Posture, 3: 13-18. 